\title{
Development of a PVP/kappa-carrageenan/PEG hydrogel dressing for wound healing applications in Sri Lanka
}

\author{
D. Awanthi De Silva ${ }^{1 *}$, Buddhika U. Hettiarachchi ${ }^{2}$ L.D.C. Nayanajith ${ }^{2}$, M.D. Yoga Milani² and \\ J.T.S. Motha ${ }^{2}$ \\ ${ }^{1}$ 47/2, Irvine Street, Gwynneville, NSW 2500, Australia. \\ ${ }^{2}$ Materials Technology Institute, Industrial Technology Institute, 363, Bauddhaloka Mawatha, Colombo 07.
}

\begin{abstract}
Research was carried out to develop a wound dressing hydrogel using polyvinylpyrrolidone (PVP), kappa carrageenan and polyethylene glycol (PEG) by $\mathrm{Co}^{60}$ gamma irradiation. The gel has a soothing effect and it is not necessary to change the dressing too often. This gel is suitable for the tropical conditions of Sri Lanka and has a comparatively long shelf-life. The cost is affordable. Aqueous solutions of PVP \& kappa-carrageenan were mixed with low molecular weight PEG to form the pseudogel which was then subjected to $25 \mathrm{kGy}$ irradiation. Absorption behaviour in distilled water, 9\% saline water and pseudoextracellular fluid, tensile properties, bioadhesion onto human skin and sterility/shelf-life were studied in comparison with commercial hydrogels. The chemical and physical behaviour of the PVP/carrageenan/PEG hydrogel were further studied by analyzing FTIR spectra and SEM images. The observations made in the study concluded that the properties of the developed hydrogel are comparable to that of the commercial hydrogels, to the level required to apply as a wound dressing. The results obtained from testing and analysis of the hydrogel further confirm that the PVP/carrageenan/PEG hydrogel has been developed to the point where it is ready to proceed to clinical trials.
\end{abstract}

Keywords: Gamma irradiation, hydrogel, kappa-carrageenan, polyethyleneglycol (PEG), polyvinylpyrrolidone (PVP), wound dressing.

\section{INTRODUCTION}

Wound healing is a dynamic process and the performance of a wound dressing can influence the progress of healing. It is widely accepted that a warm, moist environment encourages rapid healing and most modern wound care products are designed to provide these conditions (Balakrishnan et al., 2005). In Sri Lanka conventional treatments used for burn wounds include silver sulphadiazine cream, which is most commonly used due to its water solubility, spreading effect over the wound and the low cost. In addition paraffin gauze and vaseline gauze (with or without Betadine) are also used for the treatment of superficial and partial burn wounds. Acticoat, an antimicrobial barrier dressing, is also used by local doctors for partial and deep burns. This dressing consists of a silver nanocrystalline mesh and promotes moist wound healing. However, low spreadabilty, the need for the dressing to be changed frequently and the high cost prevent it from being used more widely. Among the many categories of advanced wound dressing products available today, hydrogels incorporating natural polysaccharides are popular because they are effective, comfortable, easy to use and cost effective. With proper use, these agents provide control of wound surface hydration and absorb the excess exudate and often provide moisture facilitating moist wound healing (Eisenbud et al., 2003).

Hydrogels are a class of polymer materials which forms three dimensional networks. They can absorb large amounts of liquid without dissolving and retain the liquid even under pressure. Hydrogels have been proven effective in facilitating repair of pressure, diabetic, vascular, burn and other wounds (Eisenbud et al., 2003). Aqueous solutions of synthetic polymers like polyvinylpyrrolidone (PVP) and polyethylene glycol (PEG) chemically cross-link with natural polysaccharides such as carrageenan, under suitable conditions, in the presence of initiators and cross linking agents to form gels. In this study we have

\footnotetext{
* Corresponding author (awanthi20002000@yahoo.com)
} 
used gamma irradiation since this method facilitates easy process control, the possibility of joining hydrogel formation and sterilization in one technological step, and initiators and cross linkers are not necessary which could be harmful and difficult to remove.

Polyvinyl alcohol and polyvinyl pyrrolidone (PVA (20 wt \%) -PVP $(8 \mathrm{wt} \%)\}$ blended hydrogel for wound dressing has been prepared previously(Razzaketal.,2001) with a maximum absorption rate of $250 \%$. Research work by Varshney et al. (2007) showed the possibility of making a hydrogel using PVA, carrageenan and agar while another study (Ajji et al., 2005) proved the possibility of making wound dressing hydrogel using PVP, PEG and agar. The presence of agar, however, may cause easier penetration of microorganisms into the hydrogel particularly in a tropical environment where humidity is high. Yusof et al. (2007) developed a PVP hydrogel containing Malaysian honey while Park et al. (2004) developed a PVP, PVA hydrogel consist of Aloe vera. Hydrogels from varying concentrations of PVP and kappa - carrageenan $(3 \%, 6 \%, 9 \%$, and 15\%) have been synthesized (Luculle et al., 2003). However the degree of swelling exhibited by this combination was comparatively low compared to other hydrogels.

Commercialized hydrogels of some such compositions are available in USA, European countries like UK, Poland, Switzerland and Asian countries like Japan, Korea, India, Malaysia and Indonesia, under different trade names (i.e. Convatec's Duoderm, Replicare, Aqua Gel, Kik Gel, Hizel, Geliperm, Vigilon, Ivalon). The high cost and low availability prevent wide use of these imported hydrogels in local hospitals. The present work involved the preparation of a wound/burn dressing hydrogel by $\mathrm{Co}^{60}$ gamma irradiation of PVP, kappa carrageenan and PEG which is a new combination of raw materials. PVP is a film/network forming polymer with excellent wetting properties which is used in pharmaceutical applications, while carrageenans are hydrophilic polymers extracted from red sea weed Eucheuma and composed of D-galactose units having half ester sulphate moieties linked with glycosidic linkages. PEGs are oligomers and/or polymers of ethylene oxide widely used in lubricant applications in the biomedical field. It incorporates elasticity, adhesion and tacky properties while improvng the hydrogel barrier qualities against bacteria (Ajji et al., 2005). The absorption rate, mechanical properties, bio-adhesion, and sterility of the final product were studied in comparison with commercial hydrogels. Fourier transform infrared spectroscopy (FTIR) and scanning electron microscope (SEM) images of the developed novel wound dressing hydrogel were analyzed.

\section{METHODS \& MATERIALS}

PVP with average molar mass of 1,000,000-1,500,000

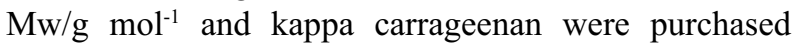
from Sigma-Aldrich. PEG of $400 \mathrm{Mw} / \mathrm{g} \mathrm{mol} \mathrm{m}^{-1}$ was supplied by Scharlou Chemie S.A., Spain. All the chemicals were used without further purification. Other chemicals such as $\mathrm{NaCl}, \mathrm{KCl}, \mathrm{NaHCO}_{3} \& \mathrm{NaH}_{2} \mathrm{PO}_{4}$ to prepare Homsy's pseudo-extracellular fluid were used as received. Distilled water was used as the solvent to prepare hydrogel samples. Polyethylene terephthalate (PET) trays ( $0.75 \mathrm{~mm}$ thick) and PET films (20 $\mu$ thick) were used to pour and cover the pseudogel samples for settling. High thickness poly ethylene pouches were used to pack the samples for irradiation.

Preparation of PVP/carrageenan/PEG hydrogel: The hydrogel dressing preparation consisted of 3 steps ;(a) pseudogel preparation (b) irradiation and (c) sterilization. $7 \%(\mathrm{w} / \mathrm{v})$ PVP, $1 \%(\mathrm{w} / \mathrm{v})$ carrageenan and $1.5 \%(\mathrm{w} / \mathrm{v})$ PEG was mixed to prepare the hydrogel composition. Aqueous solutions of the components (PVP, carrageenan and $P E G$ ) were prepared by dissolving them at appropriate temperatures (PVP by keeping overnight in distilled water, carrageenan by dissolving it with distilled water at $60{ }^{\circ} \mathrm{C}$ ). A mixture of the 3 components was kept in an oven maintained at $60-70{ }^{\circ} \mathrm{C}$, for the removal of bubbles. The mixture solution was poured into PET moulds/trays with required volumes ( $3-4 \mathrm{~mm}$ thickness), where they were kept for $1 / 2-1 \frac{1}{2} \mathrm{~h}$ at ambient temperature for proper settling (pseudogel formation). These moulds were covered with $20 \mu$ thick PET sheets and packed and sealed in polyethylene (PE) pouches, minimizing aeration. Hydrogel packages were irradiated at a dosage of $25 \mathrm{kGy}$ and a dose rate of $2.08 \mathrm{kGy} / \mathrm{h}$ using $\mathrm{Co}^{60}$ gamma irradiation. This dose of radiation is sufficient to sterilize the materials and form a stable three-dimensional network of the hydrogel which allows liquids to penetrate into the structure to a certain volume without destroying its shape. Samples of required sizes were cut from the dressing to carry out different experiments.

Determination of swelling characteristics: The samples were immersed in distilled water, 9\% saline water or pseudoextracellular fluid (PECF) solution at $40{ }^{\circ} \mathrm{C}$ in the proportion of mass of gels : volume of the liquid 1:100. Swelling was allowed to continue until a constant weight of the gel was reached. Before weighing a gel sample, excess liquid was filtered out using a $75 \mu \mathrm{m}$ sieve and the surface water was gently removed with a filter paper. Determination of swelling characteristics in distilled water, 9\% saline water and PECF solution was done in triplicate. 
(a) absorption characteristic in distilled water: This gives a measure of the water absorption behaviour of the initial irradiated hydrogel (Razzak et al., 2001).

$$
A_{w}(\%)=\frac{\left(W_{s}-W_{0}\right)}{W_{0}} \times 100 \%
$$

Where $A_{w}$ is the water absorption , $W_{s}$ is the weight of swollen gel at any time interval and $\mathrm{W}_{0}$ is the weight of initial gel sample (before being immersed in distilled water)

(b) absorption characteristics in $9 \%$ saline water: This gives a measure about the absorption behaviour of the initial irradiated hydrogel in saline water. $A_{\text {sa }}(\%)$ was measured using the same equation used for distilled water (equation 1), replacing $A_{w}(\%)$ with $A_{s a}(\%)$. Where $A_{\text {sa }}$ is the absorption in $9 \%$ saline water, $\mathrm{W}_{\mathrm{s}}$ is the weight of swollen gel at any time interval and $\mathrm{W}_{0}$ is the weight of initial gel sample (before being immersed in saline).

(c) absorption characteristics in PECF solution: To obtain a more realistic behaviour of the hydrogel as a wound dressing hydrogel when applied to human (on absorbing exudate), the swelling study was performed in Homsy's PECF solution (Sen, 2005) instead of distilled water or $9 \%$ saline water. The PECF formulation (simulated wound fluid) consists of $0.68 \mathrm{~g}$ of $\mathrm{NaCl}, 0.22 \mathrm{~g}$ of $\mathrm{KCl}, 2.5 \mathrm{~g}$ of $\mathrm{NaHCO}_{3}$ and $0.35 \mathrm{~g}$ of $\mathrm{NaH}_{2} \mathrm{PO}_{4}$ in $100 \mathrm{~mL}$ of distilled water. One gram of irradiated hydrogels were left to swell in PECF solution at $40^{\circ} \mathrm{C}$. The gel was weighed at time intervals as before to observe the swelling behaviour. $\mathrm{A}_{\mathrm{PECF}}(\%)$ was measured using the same equation used in distilled water (equation 1), replacing $A_{w}(\%)$ with $A_{P E C F}$ $(\%)$ Where $A_{\text {PECF }}$ is the absorption in PECF solution, $W_{s}$ is the weight of swollen gel at any time interval and $\mathrm{W}_{0}^{\mathrm{s}}$ is the weight of initial gel sample (before being immersed in PECF solution).

Tensile strength and \% elongation at break: Both tensile strength and \% elongation at break were determined using Tinius Olsen Universal Testing Machine (Model: $\mathrm{H} 10 \mathrm{kS}$ ) with a crosshead speed of $50 \mathrm{~mm} / \mathrm{min}$ and a load of 500 N. Dumbbell shape test specimens were cut according to the ISO 37 standard (for Rubber). The data was transferred to the computer software for evaluating stress strain curve, load at breaking point, tensile strength and \% elongation at break. The PVP/Carr/PEG hydrogel was compared with the commercial PVP hydrogel.

Bio-adhesion: Bio-adhesion test was performed to measure and evaluate the adhesion capability of the hydrogel onto the human skin by comparing with the commercial PVP hydrogel. After applying a load of $0.1 \mathrm{~N}$ on the skin for $5 \mathrm{~min}$ (Sen, 2005) on $(0.4 \times 2 \times 5 \mathrm{~cm})$ samples, the adhesion strength of the hydrogel was measured using Tinius Olsen Universal testing machine (Crosshead speed: $50 \mathrm{~mm} / \mathrm{min}$, Load $500 \mathrm{~N}$ ) in ambient conditions. The test was carried out 5 times for each sample to calculate the average bio-adhesion.

Sterility: To determine the shelf-life of the irradiated PVP/Carr/PEG hydrogel product, sterility test was performed using SLS 743:1986 (Sri Lanka standard for specification for skin cream \& lotion) \& SLS 516:2 - 1991 (general guidance for enumeration of yeast \& moulds). The sterility test was performed for hydrogel samples packed either in PE pouch with $2 \mathrm{~mm}$ thick sealing or PE pouch with $8 \mathrm{~mm}$ thick sealing at ambient temperature $\left(27{ }^{\circ} \mathrm{C} \pm 2\right)$ under refrigerator conditions $\left(4^{\circ} \mathrm{C}\right)$ and at accelerated ageing conditions $\left(40^{\circ} \mathrm{C}\right)$. The accelerated ageing was performed complying with the standard ASTM F 1980-07 (standard guide for accelerated ageing of sterile barrier systems for medical devices).

Fourier transform infrared spectroscopy (FT-IR): FTIR spectra were recorded using a ThermoNicolet (Model: NICOLET 6700) FTIR spectrophotometer and the spectra were analyzed in the mid IR region. Hydrogel samples were prepared for the analysis by drying (to remove the excess solvent) in a vacuum oven and spectra were obtained in the range of $4000-400 \mathrm{~cm}^{-1}$ during 64 scans, with $2 \mathrm{~cm}^{-1}$ resolution, using ATR mode.

Scanning electron microscopy (SEM): Standard procedure was used to prepare samples for SEM. Freeze dried samples of hydrogel were taken for SEM analysis and surface, transverse and cross sections were recorded at different magnifications at $21 \mathrm{kV}$ using LEO $1420 \mathrm{VP}$ model.

\section{RESULTS}

The absorption behaviour in distilled water of PVP/Carr/PEG gel compared to the commercial product showed a significant decrease of the absorbing ability (Figure 1). The maximum absorption of $679 \%$ was observed for PVP/Carr/PEG gel at 72 hours swelling time and after 24 hours the rate of increase gradually decreased. The commercial gel showed a significant swelling of $2313 \%$ in 6 hours but lost the consistency of the gel after breaking at maximum swelling. In $9 \%$ saline water (Figure 2) the absorbing ability drastically decreased (maximum 332\%) in the commercial gel whereas in the PVP/Carr/PEG gel the decrease occured at a comparatively low percentage (maximum 231\%). 
On the PECF solution maximum swelling was achieved for both hydrogels in a short period of immerse time (4-6 hours) and the maximum observed were 49\% and $63 \%$ for $\mathrm{PVP} / \mathrm{Carr} / \mathrm{PEG}$ gel and commercial gel respectively (Figure 3 ). The results given in Figures 1,2 and 3 correspond to the average of three readings.

The tensile strength and elongation at break of the hydrogel was compared against 2 commercial hydrogels (Table 1). The tensile strength of the PVP/Carr/PEG hydrogel showed that it has a better tensile strength $\left(509 \pm 6 \mathrm{gcm}^{-2}\right)$ than the other two hydrogels $\left(407 \mathrm{gcm}^{-2}\right)$.
The elongation at break of the PVP/Carr/PEG hydrogel was observed to be lesser $(138 \% \pm 5)$ than the values obtained for the commercial PVP and commercial PVA hydrogels (142\% and 180\% respectively). The highest value was seen in PVA gel. The bio-adhesion results for the study (Figure 4) showed that both the commercial hydrogel and the PVP/Carr/PEG hydrogel have $1 \times 10^{-3} \mathrm{~N}$ range value when applied on 2 patients ( $A$ and $B$ ). The sterility study results showed a significant improvement of shelf life when pouches with $8 \mathrm{~mm}$ sealing were used for packing. PVP/Carr/PEG hydrogels packed in $\mathrm{PE}$ pouches with $2 \mathrm{~mm}$ sealing exceeded the permissible level

Table 1: Comparison of mechanical properties of the PVP/Carrageenan/PEG hydrogel with the commercial hydrogel

\begin{tabular}{lcc}
\hline Hydrogel sample type & Tensile strength $\left(\mathrm{gcm}^{-2}\right)$ & $\%$ Elongation at break \\
\hline PVP/Carrageenan/PEG hydrogel & $509 \pm 5$ & $138 \% \pm 5$ \\
Commercial PVP hydrogel & $407 \pm 8$ & $142 \% \pm 4$ \\
Commercial PVA hydrogel & $407 \pm 6$ & $180 \% \pm 5$ \\
\hline
\end{tabular}

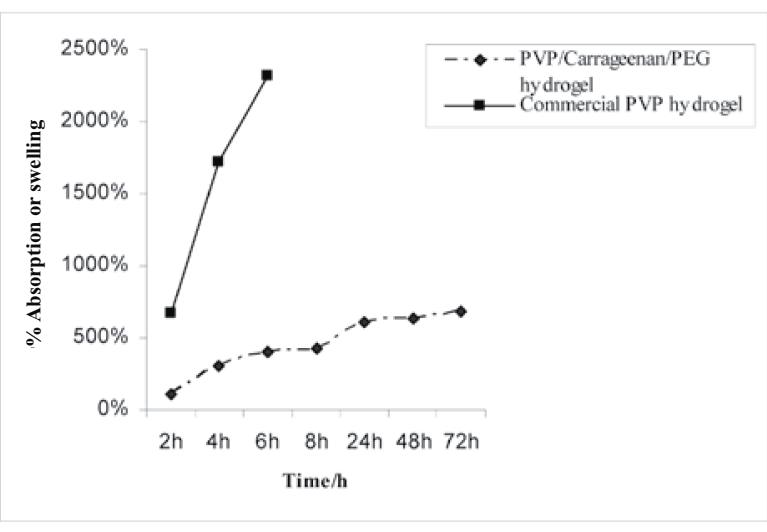

Figure 1: Absorption behaviour of PVP/Carr/PEG hydrogel in distilled water compared to the commercial PVP hydrogel

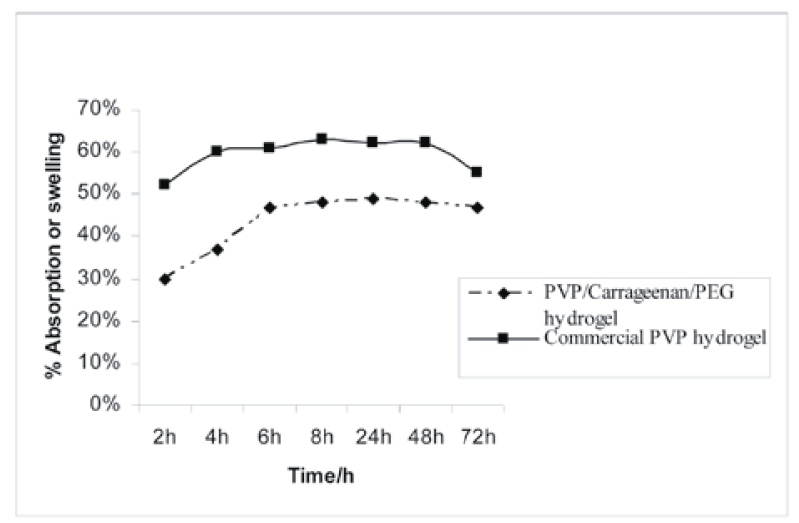

Figure 3: Absorption behaviour of PVP/Carr/PEG hydrogel in PECF compared to the commercial PVP hydrogel

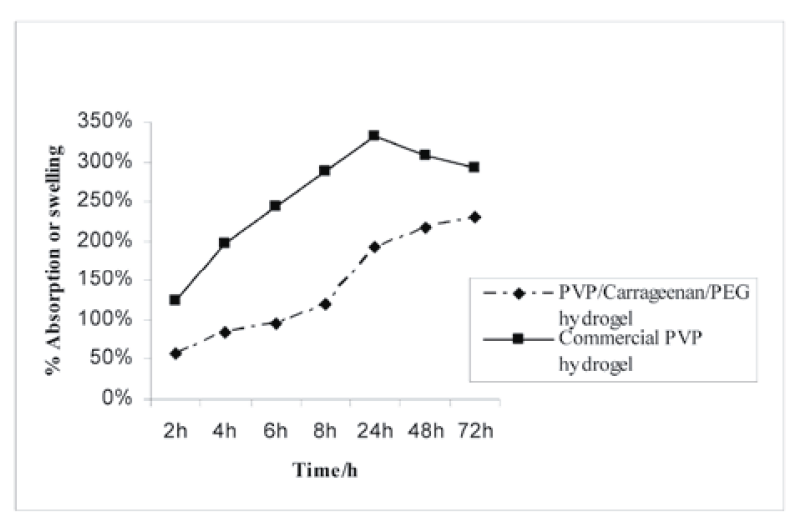

Figure 2: Absorption behaviour of PVP/Carr/PEG hydrogel in 9\% saline water compared to the commercial PVP hydrogel

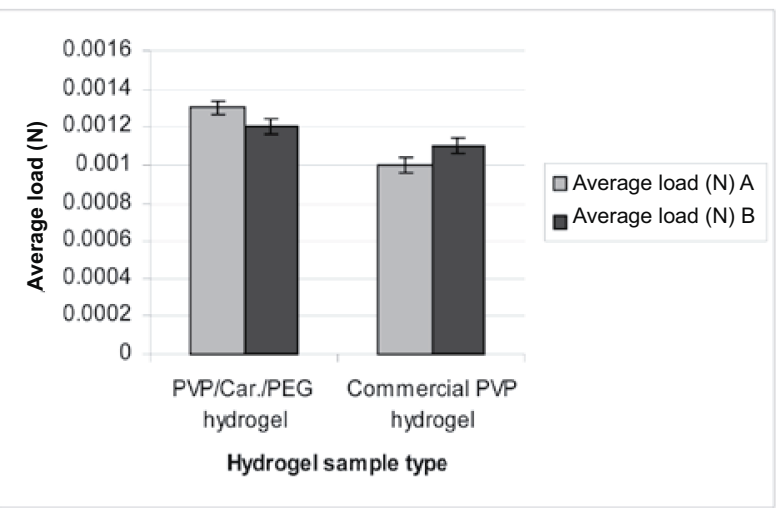

Figure 4: Average load $(\mathrm{N})$ of bio-adhesion onto the human skin (A) person 1 (B) person 2 
of bacterial and yeast colonies after 1 month of storage, under ambient temperature, refrigerator conditions and accelerated ageing conditions at $40^{\circ} \mathrm{C}$ (Table 2). For hydrogels packed in PE pouches with $8 \mathrm{~mm}$ sealing no bacterial or yeast growth was detected under all three conditions upto 6 months (Table 3 ) as per the SLS standards 743:1986 and 516-2:1991 respectively.

FTIR spectra of pure PVP, pure carrageenan, pure $\mathrm{PEG}$ and $\mathrm{PVP} / \mathrm{Carr} / \mathrm{PEG}$ hydrogels are given in Figure 5. The major peak assignments of PVP, carrageenan and PEG are summarized in Table 4. The FTIR spectrum of PVP/Carr/PEG hydrogel consisted of the functional groups present in all three pure components. $\mathrm{CH}_{2}$ stretch, contribution from $\mathrm{C}=\mathrm{O}$ and $\mathrm{N}-\mathrm{C}$ in pure PVP, $\mathrm{O}-\mathrm{H}$ (stretch), sulfates (stretch), glycosidic linkage, $\mathrm{C}_{4}-\mathrm{O}-\mathrm{S}$ in galactose of carrageenan and $\mathrm{C}-\mathrm{O}-\mathrm{C}$ (stretch) in PEG was clearly observed in the FTIR spectrum for irradiated PVP/Carr/PEG hydrogel (Figure 5). When the FTIR spectrum of PVP/Carr/PEG pseudogel (before irradiation) was compared with the irradiated hydrogel there was no significant difference in peak patterns or the peak positions (Figure 6).

SEM images of the vacuum dried PVP/Carr/PEG pseudogel and the hydrogel are illustrated in Figure 7. The magnified images (approx $150 \mathrm{x}$.) of the top surfaces of the two gels showed a clearly distinguishable difference. The vacuum dried surface of the pseudogel exhibited cracks in some places [Figure 7(a)] while the irradiated hydrogel [Figure 7(b)] did not show any such cracks throughout the surface area. SEM images of Figure 7 were recorded after freeze drying of the hydrogel samples instead of vacuum drying. Top surface, transverse section and cross section were recorded in Figure 8, with $150 \mathrm{x}$, $90 \mathrm{x}$ and $60 \mathrm{x}$ magnifications respectively.

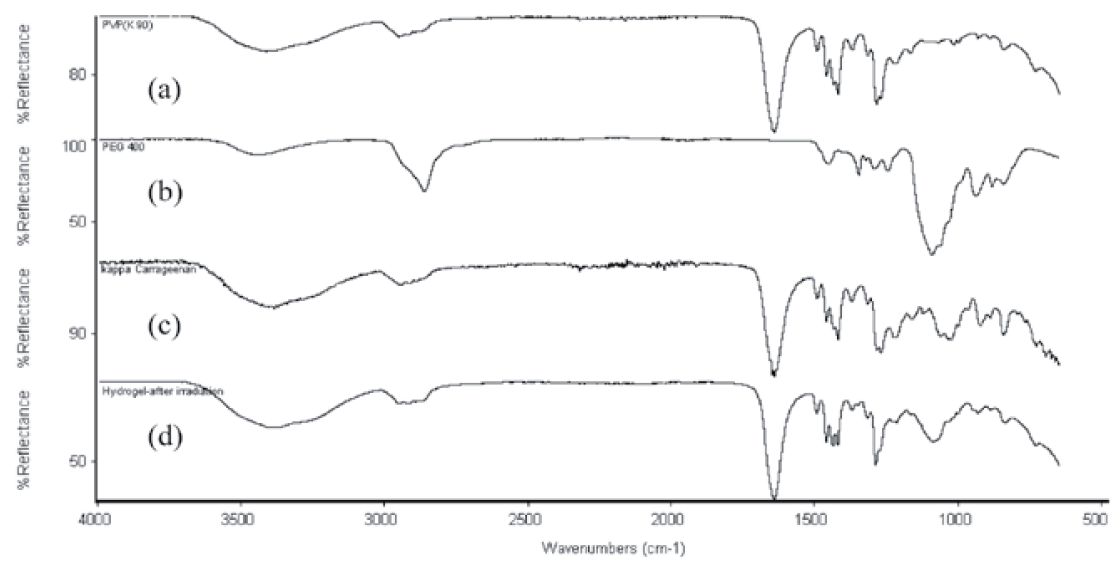

Figure 5: FTIR spectra of (a) pure PVP, (b) pure PEG, (c) pure kappa carrageenan and (d) PVP/Carr/PEG hydrogel

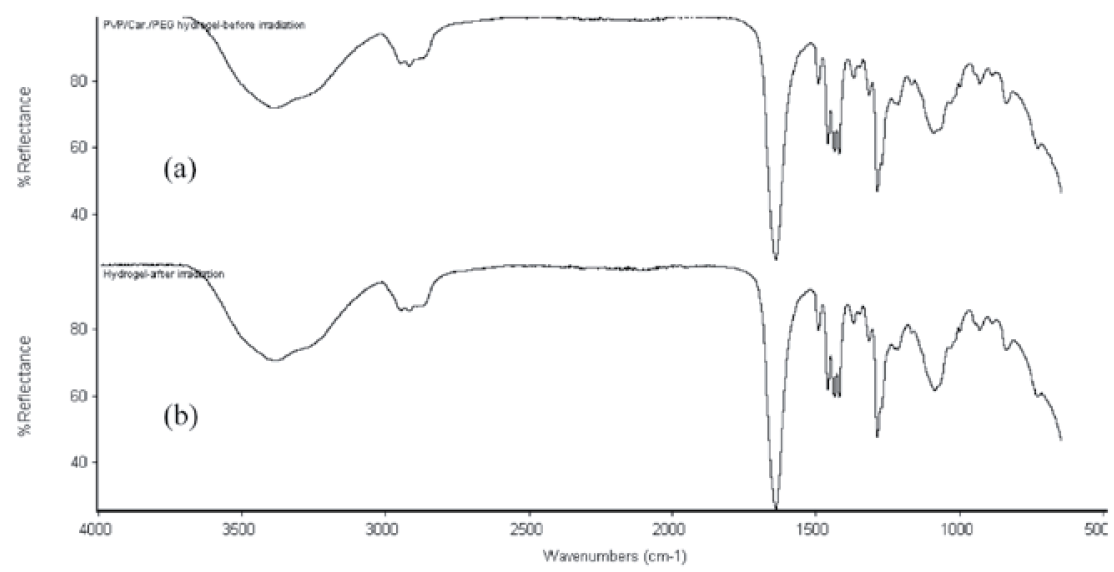

Figure 6: FTIR spectra of (a) PVP/Carr/PEG pseudogel (before irradiation) and (b) PVP/Carr/PEG hydrogel (after irradiation) 
Table 2: Sterility behaviour of PVP/carrageenan/PEG hydrogel when packed in LDPE pouches with $2 \mathrm{~mm}$ sealing

\begin{tabular}{llccccc}
\hline & \multicolumn{5}{c}{ Low thickness PE pouch with $2 \mathrm{~mm}$ sealing } \\
& \multicolumn{3}{c}{ Soon after irradiation } & & 1 month \\
\cline { 2 - 7 } & $\begin{array}{c}\text { Ambient } \\
\text { conditions }\end{array}$ & $\begin{array}{c}\text { Refrigerator } \\
\text { conditions } \\
\left(4{ }^{\circ} \mathrm{C}\right)\end{array}$ & $\begin{array}{c}\text { Accelerated } \\
\text { ageing } \\
\left(40^{\circ} \mathrm{C}\right)\end{array}$ & $\begin{array}{c}\text { Ambient } \\
\text { conditions }\end{array}$ & $\begin{array}{c}\text { Refrigerator } \\
\text { conditions } \\
\left(4{ }^{\circ} \mathrm{C}\right)\end{array}$ & $\begin{array}{c}\text { Accelerated } \\
\text { ageing } \\
\left(40^{\circ} \mathrm{C}\right)\end{array}$ \\
\hline Bacterial count/g & $<10$ & $<10$ & $<10$ & $4.7 \times 10^{2}$ & $1.3 \times 10^{3}$ & $6.3 \times 10^{3}$ \\
Pseudomonas aeruginosa count/10 g & Absent & Absent & Absent & Absent & Absent & Absent \\
Staphylcoccus aureus count/10 g & Absent & Absent & Absent & Absent & Absent & Absent \\
Yeast \& mould count/g & 10 & 10 & 10 & $9.8 \times 10^{3}$ & $4.1 \times 10^{3}$ & $1.2 \times 10^{4}$ \\
\hline
\end{tabular}

Table 3: Sterility behaviour of PVP/carrageenan/PEG hydrogel when packed in HDPE pouches with $8 \mathrm{~mm}$ sealing

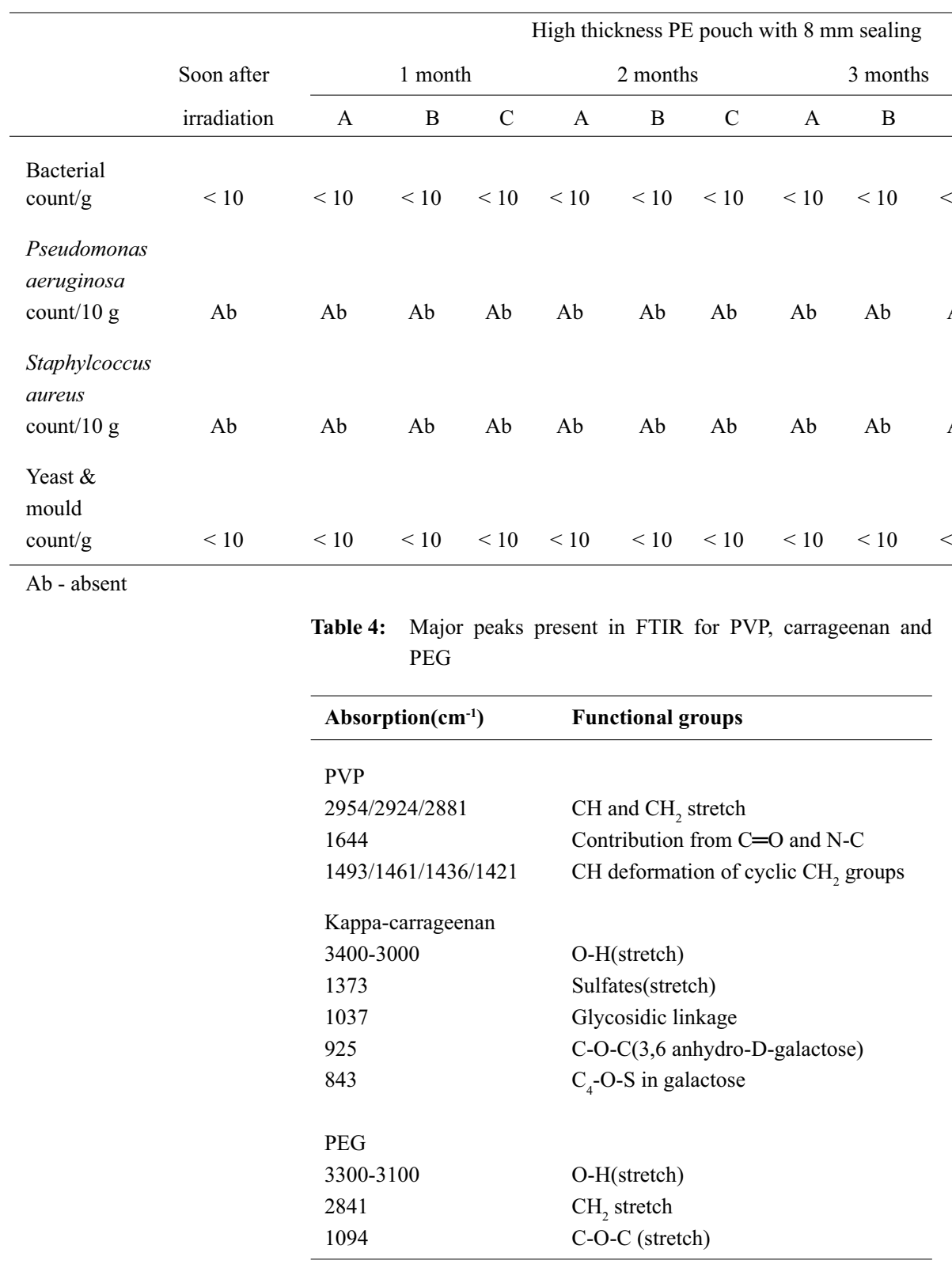




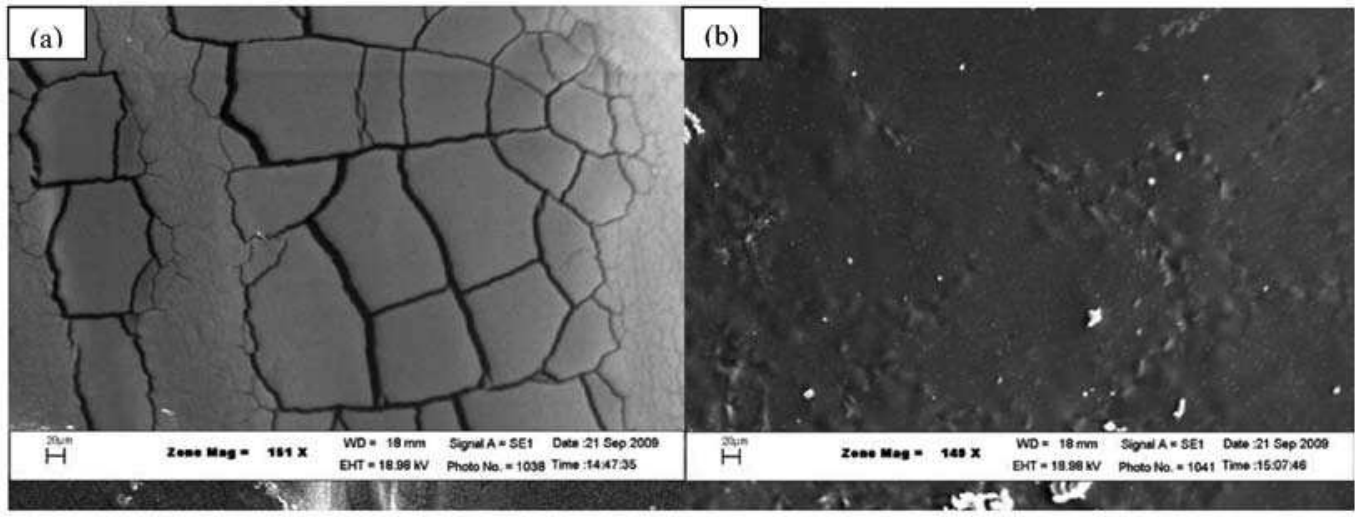

Figure 7: SEM images of the top surface of vacuum dried (a) pseudogel and (b) irradiated gel of PVP/Carrr/PEG hydrogel

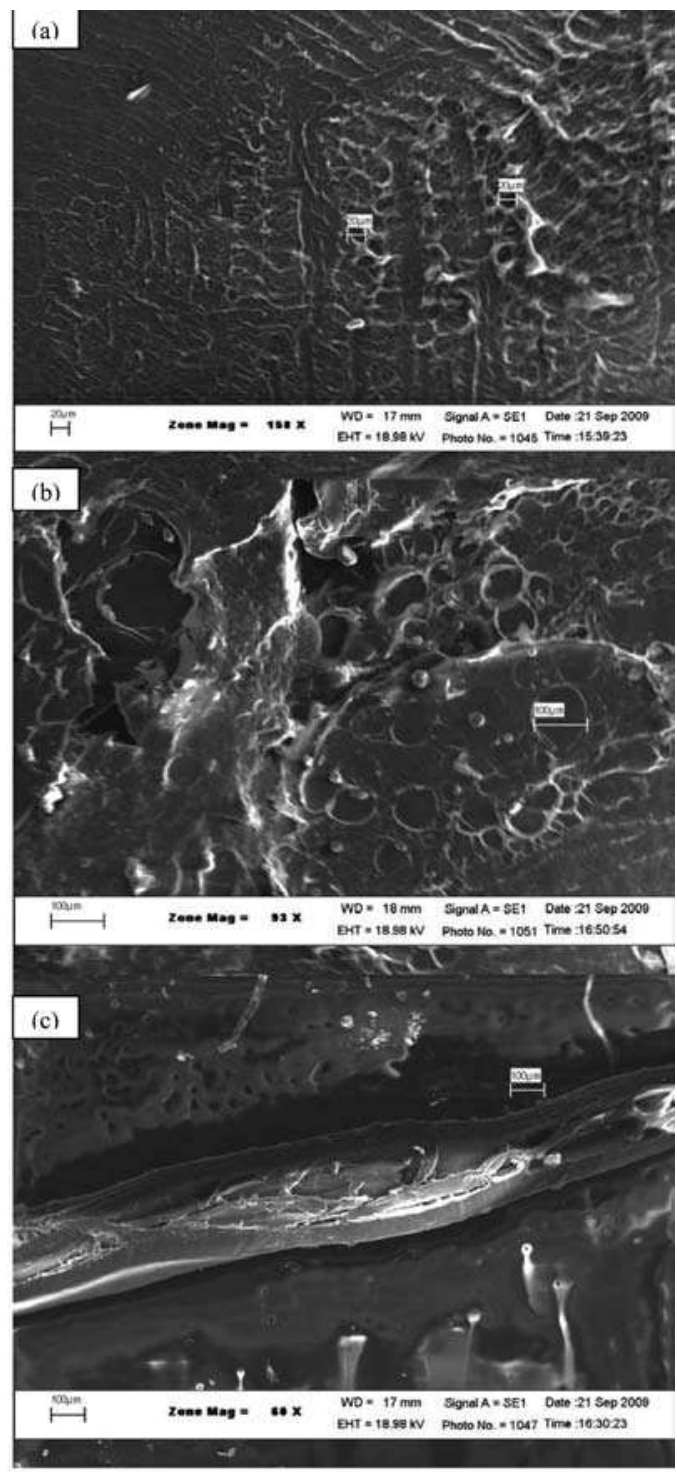

Figure 8: SEM images of the freeze dried PVP/Carr/PEG hydrogel (a) top surface (b) transverse section and (c) cross section

\section{DISCUSSION \& CONCLUSION}

The swelling results for both hydrogels indicate that there is a significant decrease in the ability to absorb when the ionic strength of the absorbing liquid increases.

When the hydrogels in distilled water were compared with the hydrogels in PECF, the maximum absorbing ability decreased 35 fold for the commercial gel and 14 fold for the PVP/Carr/PEG hydrogel, approximately. The performance of the hydrogel could be further improved as a wound dressing hydrogel if the ionic nature of the hydrogel could be increased. It is stated in literature that kappa-carrageenan is a polyanion and therefore salts affect its gelation behaviour (Sen, 2005). Therefore it is possible to enhance the ionic nature of the hydrogel by incorporating/grafting ionic materials such as salts into the hydrogel network. Since the PVP/Carr/PEG gel did not break or dissolve as in the commercial gel even after 72 hours of swelling, it can be suggested that the PVP/Carr/PEG gel could be applied for a longer period of time for wounds, thereby avoiding the necessity to change the dressing frequently.

The mechanical properties such as tensile strength and elongation at break of the hydrogel are determined by the crosslink and/or inter-penetrating network (IPN) density (Lucille et al., 2003; Sen, 2005). The results show that the PVP/Carr/PEG hydrogel has a higher crosslinking/IPN density compared to other commercial hydrogels (Table 1). These properties are important since the hydrogel dressing has to be applied on contour areas in the body. It is a known fact that the dressing is more patient friendly if it could be applied and removed from the wound without breaking into pieces. Since the PVP/Carr/PEG has a higher tensile strength and lower elongation at break, it could be introduced as a more patient friendly dressing to the market. 
Bio-adhesion is generally defined as the ability of the biological or synthetic material to stick to the skin or a mucous membrane. Many adhesive dressings cause secondary injury and pain when peeled off from wounds. $\mathrm{PVP} / \mathrm{PEG}$ blends were reffered to in the literature as pressure sensitive adhesives (Sen, 2005). Since the human body surface is rough in average, the softness of the hydrogel is an added advantage for the soothing effect of the patient. Though the PVP/Carr/PEG hydrogel showed similar bio-adhesion to the human skin as in the commercial hydrogel, the adhesion energy could be further reduced. This can be attained by decreasing the irradiation dose (Sen, 2005) and also by a future study on varying PEG concentrations with different PEG molecular weights.

The shelf-life of the hydrogel product is heavily dependant on the packaging method. Even though the product is sterilized during the crosslinking of the product simultaneously, when the packing method allows the gel to come in contact with air, Pseudomonas aeruginosa, Staphylcoccus aureus and yeast and mould counts were observed exceeding the permissible level even before 1 month of storage. This is a critical issue that should be addressed specially to protect the wound from further infection. The microbial growth was greatly reduced by minimizing the aeration inside the product before subjecting to gamma irradiation.

A previous study (Lucille et al., 2003) proposed that carrageenan graft to PVP even at a low dose of $5 \mathrm{kGy}$. In the present study $25 \mathrm{kGy}$ was used as an irradiation dose, since it has an advantage of cross linking and sterilizing the product in one step (Ajji et al., 2005). FTIR results suggests that both carrageenan and PEG grafts to PVP after irradiation forms an interpenetrating network(IPN) (Lucille et al., 2003; Sen, 2005) while in carrageenan and PEG chains are entangled within the PVP network. These results could be further explained using published literature (Schacht, 2004). It was shown that the synthesis of hydrogels using synthetic polymers are obtained via three methods, (a) polymerization of hydrophilic monomers (i.e. methacrylates, methacrylamides), (b) polymerization of low molecular weight prepolymers/ oligomers (i.e. PEG) and/or (c) polymerization of polymers (i.e. PVP, polyvinyl alcohol). He had also identified gel formation by helix formation, association of helices and formation of junction zones upon cooling in aqueous solution as the method of hydrogel formation in natural polysaccharides (i.e. agar, carrageenan, carboxymethylcellulose). The results also depict that even though natural biopolymers tend to degrade upon irradiation, the $25 \mathrm{kGy}$ dosage was withstood by carrageenan and promoted crosslinking over degradation.
The comparison between the pseudogel and the hydrogel (Figure 6) spectra showed a slight shift of O-H (stretch) from $3384 \mathrm{~cm}^{-1}$ to $3376 \mathrm{~cm}^{-1}$ and $\mathrm{CH}, \mathrm{CH}_{2}$ (stretch) from $2921 \mathrm{~cm}^{-1}$ to $2919 \mathrm{~cm}^{-1}$ respectively, where all the other functional peaks remained unchanged. Based on this result it is proposed that even though chemical bonds could have been formed on irradiation via $\mathrm{OH}, \mathrm{CH}$ and $\mathrm{CH}_{2}$ functional groups of PVP, PEG and carrageenan, since most of the functional groups remained unchanged during irradiation, formation of interpenetrating network might be the prominent method of network formation over chemical crosslinking.

The SEM images in Figure 7 (a) and 7 (b) clearly depict the effect of gamma irradiation on pseudogel. The weak temporary bonds formed during the formation of pseudogel (which was broken during the vacuum drying process) have formed more strong chemical cross links and IPN while irradiation (which withstood the vacuum drying conditions). When the images of vacuum dried and freeze dried gels were compared with each other, it also showed that the freeze dried gels gave better images than vacuum dried gels pointing to freeze drying as a method of selection, for hydrogel sample preparation for SEM. The images in Figure 8 show the porous structure/ crosslinked network of the PVP/Carr/PEG hydrogel. The pore sizes were within the micrometer $(\mu \mathrm{m})$ range as observed in the transverse and cross sections. But the effective pore sizes would be much smaller due to the overlapping of pores within the network (Varshney, 2007). The pore size of the network has a direct effect on the absorbing/swelling abilities of the hydrogel and it also gives an idea of the degree of crosslinking. With the decreasing of pore diameter, the degree of crosslinking increases where it increases the strength of the gel. It also affects the absorbing ability in a positive way, where the smaller pores will increase the surface/area ratio to absorb more and retain it even under pressure, which is an essential quality of an absorbing wound dressing.

As an overall conclusion of the laboratory trials being conducted on developing a wound dressing hydrogel using PVP, carrageenan and PEG, it is concluded that the product is suitable and ready for performance of clinical trials using human patients. The results have shown some improved properties than the commercialized products as well as some defects that have to be addressed in developing an ideal wound dressing. The mechanical properties, shelf-life and the sterility properties gave satisfactory results while some improvements could be made on absorption characteristics. The swelling/ absorption characteristics should be improved by increasing the ionic nature of the gel to apply on noninfectious wounds. The FTIR data confirmed that the 
formation of three-dimensional network (during gamma irradiation) occurred by interpenetrating network formation than by chemical crosslinking.

\section{Acknowledgement}

The author/s thank Ms. Samantha Kulatunge, Head/ Radiation Protection Division, Atomic Energy Authority for providing irradiation facilities together with scientific and technical guidance and Dr Iresha Kottegoda, Senior Research Officer/ Materials Technology Section, Industrial Technology Institute for her invaluable support and guidance to make this research a success.

\section{References}

1. Ajji Z., Othman I. \& Rosiak J.M. (2005). Production of hydrogel wound dressings using gamma irradiation. Nuclear Instruments \& Methods in Physics Research Section B: Beam Interactions with Materials \& Atoms 229(3-4):375-380.

2. Balakrishnan B., Mohanty M., Umashankar P.R. \& Jayakrishnan A. (2005). Evaluation of an in situ forming hydrogel wound dressing based on oxidized alginate and gelatin. Biomaterials 26(32):6335-6342.

3. Eisenbud D., Hunter H., Kessler L. \& Zulkowski K.(2003). Hydrogel wound dressings: where do we stand in 2003 ? Ostomy/Wound Management 49 (10):52-57.
4. Lucille A.V., Relleve L.S., Aranilla C.T. \& Rosa M.D. (2003). Properties of radiation synthesized PVP-kappa carrageenen hydrogel blends. Radiation Physics \& Chemistry 68(5):901-908.

5. Park K.R., Nho Y.C. \& Kang P.H. (2004). Preparation and characterization of PVA/PVP /Aloe vera hydrogels by irradiation. Journal of Applied Polymer Science 91(3): 1612-1618.

6. Razzak M.T., Darwis D., Zainuddin \& Sukirno (2001). Irradiation of polyvinyl alcohol and polyvinyl pyrrolidone blended hydrogel for wound dressing. Radiation Physics \& Chemistry 62(1):107-113.

7. Schacht E.H. (2004). Polymer chemistry and hydrogel systems. Journal of Physics: Conference Series 3: Third International Conference on Radiotherapy Gel Dosimetry, pp.22-28, Institute of Physics Publishing, Belgium.

8. Sen M. \& Avci E.N.(2005). Radiation synthesis of poly (N-vinyl-2-pyrrolidone) k-carrageenan hydrogels and their use in wound dressing applications.1.preliminery laboratory tests. Journal of Biomedical Materials Research 74 A(2): 187-196.

9. Varshney L. (2007). Role of natural polysaccharides in radiation formation of PVA-hydrogel wound dressing. Nuclear Instruments \& Methods in Physics Research Section B: Beam Interactions with Materials \& Atoms 255(2):343-349.

10. Yusof N., Hafiza A.H.A., Zohdi R.M. \& Baker M.Z. (2007). Development of honey hydrogel dressing for enhanced wound healing. Radiation Physics \& Chemistry 76(11-12): 1767 - 1770 . 